\title{
Hydrogen-induced buckling of Pd films deposited on various substrates
}

\author{
Marián Vlček ${ }^{1, a}$, František Lukáć ${ }^{1, b}$, Martin Vlach ${ }^{1, c}$, Ivan Procházka ${ }^{1, d}$, Stefan \\ Wagner $^{2, \mathrm{e}}$, Helmut Uchida ${ }^{2, \mathrm{f}}$, Astrid Pundt ${ }^{2, \mathrm{~h}}$, Ryota Gemma ${ }^{3, \mathrm{~g}}$, Jakub Čižek ${ }^{1, \mathrm{i}}$ \\ ${ }^{1}$ Faculty of Mathematics and Physics, Charles University in Prague, V Holešovičkách 2, CZ-180 \\ 00, Praha 8, Czech Republic \\ ${ }^{2}$ Institute of Materials Physics, University of Göttingen, Friedrich-Hund-Platz 1, D-37077 Göttingen, \\ Germany \\ ${ }^{3}$ Physical Sciences and Engineering Division, King Abdullah University of Science and Technology \\ (KAUST), Thuwal 23955-6900, Kingdom of Saudi Arabia \\ amarian.vlcek@mff.cuni.cz, bfrantisek.lukac@mff.cuni.cz, ${ }^{\mathrm{c}}$ martin.vlach@mff.cuni.cz, \\ divan.prochazka@mff.cuni.cz, ${ }^{\mathrm{e}}$ swagner@ump.gwdg.de, 'huchida@ump.gwdg.de, \\ grgemma@ump.gwdg.de, hapundt@ump.gwdg.de, jakub.cizek@mff.cuni.cz
}

Keywords: hydrogen, palladium, thin films

\begin{abstract}
Pd-H system is a model system suitable for studying interactions of hydrogen with metals. In the present work, we studied hydrogen-induced buckling of thin Pd films deposited on various substrates with different bonding strengths (sapphire, glimmer) and also the effect of deposition temperature. Lattice expansion and phase transitions were investigated by X-ray diffraction of synchrotron radiation. The influence of substrate and microstructure of the film on the buckling process and phase transformation to palladium hydride are discussed.
\end{abstract}

\section{Introduction}

Hydrogen loading causes a significant volume expansion of the metal lattice, which is isotropic in free-standing poly-crystalline bulk materials with cubic symmetry. Contrary to bulk samples, thin films are clamped to substrates which suppress the in-plane expansion. As a consequence, expansion of a thin film loaded with hydrogen is strongly anisotropic and bi-axial compressive inplane stresses are introduced into the sample. The hydrogen-induced stresses grow with increasing concentration of absorbed hydrogen and may reach the GPa range [1]. When the stored elastic strain energy overcomes the adhesion energy, local or complete detachment of the film from the substrate may be energetically favorable and buckling of hydrogen-loaded film occurs [2][3]. The buckling process itself significantly increases the dislocation density [4].

$\mathrm{Pd}$ easily adsorbs hydrogen, forms a hydride at room temperature and shows a simple phase diagram [5]. Due to these reasons Pd is often used as model system for investigations of hydrogenmetal interactions. In bulk $\mathrm{Pd}$ ( $\alpha$ phase), maximum solubility of hydrogen at $25{ }^{\circ} \mathrm{C}$, expressed as $\mathrm{H} / \mathrm{Pd}$ ratio, is $\mathrm{c}_{\mathrm{H}}=0.017$, while single phase Pd hydride ( $\alpha^{\prime}$ phase) exists at $\mathrm{c}_{\mathrm{H}}>0.60$ [6]. A two phase field, where both $\alpha$ and $\alpha^{\prime}$ phase coexist, is observed for nominal concentrations between these values. However, mechanical stresses and microstructure of Pd films significantly affect the range of hydrogen concentration where the two phase field occurs. Furthermore, solely thinness of the film limits the availability of stress relief mechanisms in very thin Pd films clamped to substrates [7].

\section{What is the aim of this paper?}

In this paper we intent to study the hydrogen loading behavior, the hydride growth and the stress release of three different types of $500 \mathrm{~nm}$ Pd films. 


\section{Experimental details}

Pd films with thickness of $500 \mathrm{~nm}$ were prepared by cold cathode beam sputtering in an ultra-high vacuum chamber with a base pressure of $10^{-10}$ mbar. Argon with pressure of $2 \times 10^{-4}$ mbar was used as a sputtering gas. Epitaxial Pd films were deposited on sapphire $(11 \overline{2} 0)$ substrates and glimmer substrates at $500{ }^{\circ} \mathrm{C}$. Nano-crystalline type films were grown on sapphire $(11 \overline{2} 0)$ substrates at room temperature. The nano-crystalline films consist of column like grains with a mean width of $50 \mathrm{~nm}$ and exhibits a fiber texture with $(111)_{P d}$ planes parallel to the substrate and random lateral orientation of the crystallites [8]. Epitaxial films grow with $(111)_{\mathrm{Pd}}$ planes parallel to the substrate surface and a well defined lateral orientation of the (111) $)_{\operatorname{Pd}}$ planes with respect to the substrate [8].

The Pd films were electrochemically charged with hydrogen in a specially designed loading cell filled with electrolyte consisting of phosphoric acid (85\%) and glycerin $(85 \%)$ mixed in the volume ratio 1:2. Constant current pulses were applied between the Pt counter electrode (anode) and the loaded sample (cathode). The hydrogen concentration introduced into the sample was calculated from the transported charge using the Faraday's law [9]. An inert protective atmosphere was used to prevent hydrogen loss by reaction with atmospheric oxygen. However, hydrogen loss occurs by creation of hydrogen bubbles during loading to high hydrogen concentrations. This leads to overestimation of the hydrogen content in the samples. Hence, even though Pd films were loaded to nominal concentrations $\mathrm{c}_{\mathrm{H}}>1.0 \mathrm{H} / \mathrm{Pd}$, actual concentration of hydrogen in the samples was always below $\mathrm{c}_{\mathrm{H}}=1.0 \mathrm{H} / \mathrm{Pd}$. Highest achieved concentrations in the Pd films were most likely around $\mathrm{c}_{\mathrm{H}}=$ $0.8 \mathrm{H} / \mathrm{Pd}[10]$.

The X-ray diffraction (XRD) studies of Pd films loaded with hydrogen were performed at the European Synchrotron Radiation Facility, Grenoble, at beamline BM20. The XRD patterns were recorded in the symmetrical Bragg-Brentano geometry using synchrotron radiation with a wavelength of $1.078 \AA$. High intensity of synchrotron radiation enabled in-situ measurement of diffraction peaks for $\alpha$ and $\alpha^{\prime}$ phase in the films loaded with hydrogen.

\section{Results}

The results of in-situ XRD investigations on hydrogen loaded nanocrystalline Pd films deposited on the sapphire substrates are shown in Figs. 1 and 2. The development of the (111) diffraction peak is plotted in Fig. 1a, for selected hydrogen concentrations. The positions of $(111)_{\alpha}$ and $(111)_{\alpha^{\prime}}$ peaks obtained from fitting of the XRD profiles are plotted in Fig $1 \mathrm{~b}$. The $(111)_{\alpha}$ peak of the $\alpha$ phase firstly shifts to lower diffraction angles due to out-of-plane expansion of the film caused by absorbed hydrogen. A shift of the peak position and a significant increase of the peak width around $\mathrm{c}_{\mathrm{H}} \sim 0.08 \mathrm{H} / \mathrm{Pd}$ indicate first stress release in the film. An interruption of the measurement by a beam loss at hydrogen content $\mathrm{c}_{\mathrm{H}}=0.20 \mathrm{H} / \mathrm{Pd}$ creates a jump in the position of $(111)_{\alpha}$ peak development. This jump is related to a waiting time of $60 \mathrm{~min}$. Within this time span, stress relaxation occurred in the film and first buckles were observed.

At higher hydrogen concentrations the $\alpha$ phase transforms into the $\alpha^{\prime}$ phase. The (111) $\alpha^{\prime}$ peak of the $\alpha^{\prime}$ phase is first detected at $\mathrm{c}_{\mathrm{H}}=0.40 \mathrm{H} / \mathrm{Pd}$ and its intensity increases with increasing hydrogen concentration while the $(111)_{\alpha}$ peak gradually disappears. The width of the $(111)_{\alpha}$ diffraction peak, shown in Fig. 2a, increases with increasing hydrogen concentration except of a short interval just before formation of the $\alpha^{\prime}$ phase. The width of the $(111)_{\alpha^{\prime}}$ peak is approximately constant with one exception at $\mathrm{c}_{\mathrm{H}}=0.40 \mathrm{H} / \mathrm{Pd}$. This is most likely caused by too weak peak intensity. The out-of-plane lattice constant for the $\alpha$ and $\alpha^{\prime}$ phase calculated from the peak positions obtained from fitting is plotted in Fig. 2b. The out-of-plane lattice constant determined for the $\alpha$ phase is higher than the corresponding lattice parameter for the bulk Pd sample [6] indicated by a dashed line in the figure. This testifies to compressive in-plane stress imposed by lattice mismatch between the films and the substrates. On the other hand, the out-of-plane lattice parameter for the $\alpha^{\prime}$ phase is lower than that for the bulk $\mathrm{PdH}_{0.6}$. During the phase transition from $\alpha$ phase to $\alpha^{\prime}$ phase the lattice parameter for the $\beta$ phase remains approximately constant while the lattice constant for 
the $\alpha$ phase slightly increases. Then the whole film was found to be buckled after finishing the hydrogen loading sequence. This testifies that hydrogen-induced stresses developing during hydrogen charging exceeded the film adhesion to the sapphire substrate.
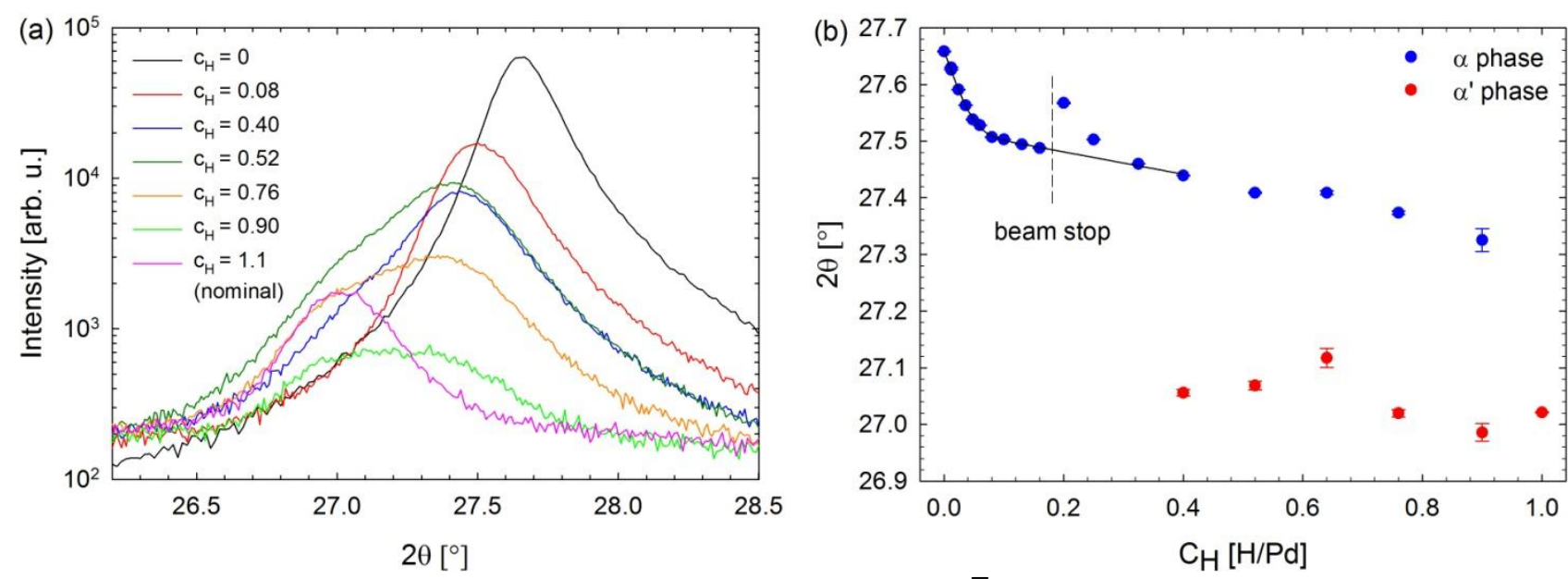

Figure 1 Nanocrystalline Pd film deposited on sapphire (112̄0) substrate: (a) the development of (111) peak for selected hydrogen concentrations; (b) the dependence of the peak position on hydrogen concentration. The lines are just to guide the eye.
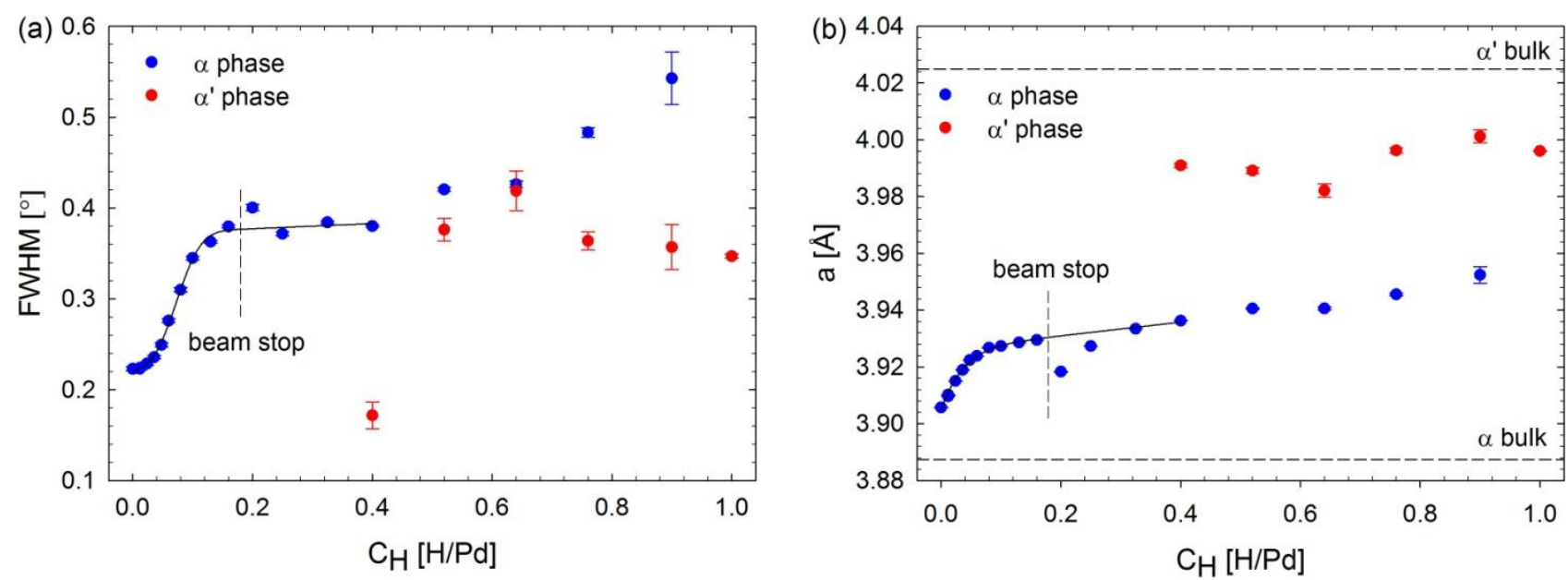

Figure 2 Nanocrystalline Pd film deposited on sapphire (112̄0) substrate: the development of (a) peak width and (b) lattice parameter during hydrogen loading. Dashed lines in the right panel shows the lattice parameter for the bulk $\alpha$ and $\beta$ phase reported in literature [6]. The lines are just to guide the eye.

The development of the (111) diffraction peak for the epitaxial film deposited on the sapphire substrate is shown in Fig. 3a for selected hydrogen concentrations. In the virgin film, the (111) $\alpha$ peak is clearly significantly narrower compared to the nanocrystalline film of Fig. 1 and 2, and the peak-to-background ratio is also substantially higher. At low hydrogen concentrations $\mathrm{c}_{\mathrm{H}}<0.04$ $\mathrm{H} / \mathrm{Pd}$, the $(111)_{\alpha}$ reflection again shifts to lower diffraction angles due to the hydrogen-induced volume expansion. At $\mathrm{c}_{\mathrm{H}}=0.04 \mathrm{H} / \mathrm{Pd}$ the $(111)_{\alpha^{\prime}}$ peak becomes visible and its intensity gradually increases with increasing hydrogen concentration while the intensity of the $(111)_{\alpha}$ peak decreases. However, it does not disappear even after loading to nominal concentrations ' $\mathrm{c}_{\mathrm{H}}=2.5 \mathrm{H} / \mathrm{Pd}^{\prime}$. The position and width of the peaks obtained from fitting are plotted in Figs. $3 \mathrm{~b}$ and $4 \mathrm{a}$, respectively. Points representing nominal concentrations higher than $\mathrm{c}_{\mathrm{H}}>1.0 \mathrm{H} / \mathrm{Pd}$ were shifted and stacked at $\mathrm{c}_{\mathrm{H}}$ $=1.0 \mathrm{H} / \mathrm{Pd}$. The shift of the $(111)_{\alpha}$ peak to lower diffraction angles at the beginning of the hydrogen loading can be clearly seen. The position of $(111)_{\alpha^{\prime}}$ peak changes to lower diffraction angles during first few loading steps after its appearance and then stays constant. The shift of the $(111)_{\alpha^{\prime}}$ peak is 
accompanied by a significant decrease of its width while the width of the $(111)_{\alpha}$ peak changes only slightly.

The development of the lattice constants for the $\alpha$ and $\alpha^{\prime}$ phase is shown in Fig. 4b. The virgin film suffers from a tensile in-plane stress demonstrated by the out-of-plane lattice parameter lower than that of bulk Pd. The $\alpha$-phase lattice parameter for the hydrogen loaded film increases and reaches the value for bulk $\mathrm{Pd}$ at $\mathrm{c}_{\mathrm{H}}=0.06 \mathrm{H} / \mathrm{Pd}$, see Fig. $4 \mathrm{~b}$, and then remains virtually constant during further loading. The $\alpha^{\prime}$ phase exhibits a lattice constant lower than the bulk value when it initially appears. During further hydrogen loading the $\alpha^{\prime}$ phase lattice parameter firstly increases and stays constant with a value lower than the bulk value. The epitaxial film remained clamped to the sapphire substrate without any buckling even at nominal hydrogen concentration ' $\mathrm{c}_{\mathrm{H}}=2.5$ $\mathrm{H} / \mathrm{Pd}^{\prime}$. This verifies a strong adhesion to the substrate for this type of films.
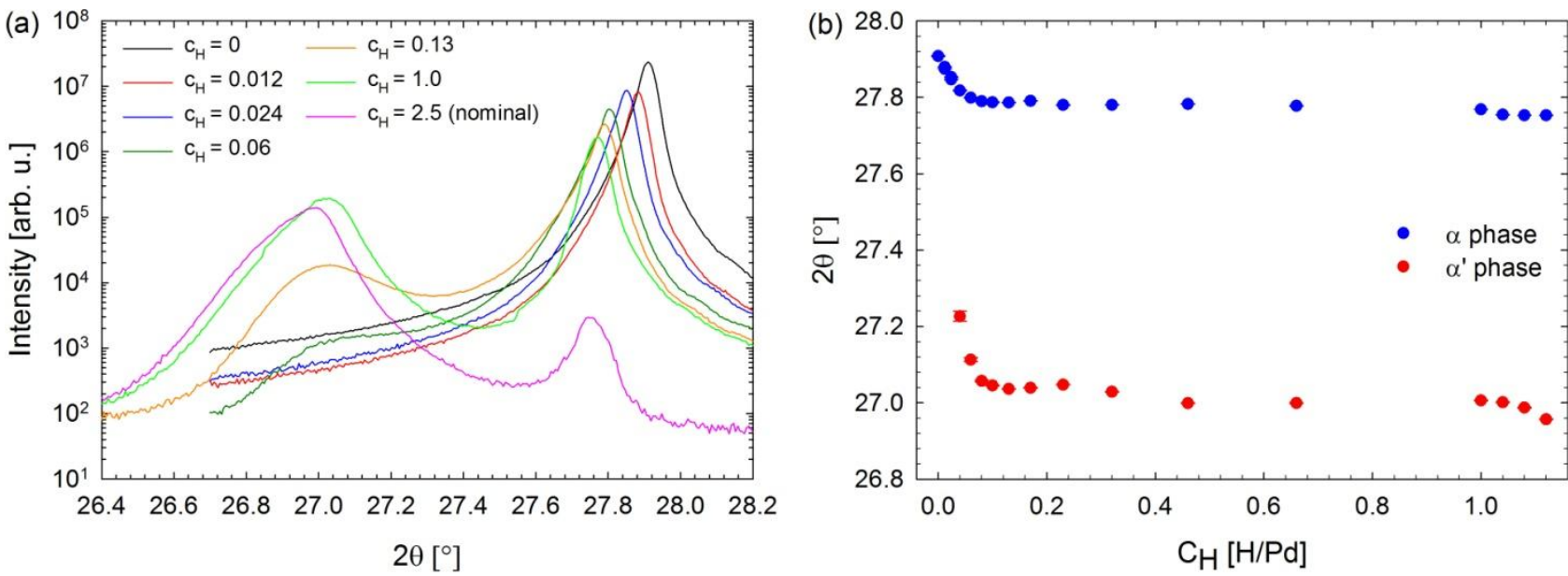

Figure 3 Epitaxial Pd film deposited on sapphire (112̄0) substrate: (a) the development of (111) peak for selected hydrogen concentrations; (b) the dependence of the peak position on hydrogen concentration. Data points grouped at $\mathrm{c}_{\mathrm{H}}=1.0 \mathrm{H} / \mathrm{Pd}$ represent nominal concentrations $\mathrm{c}_{\mathrm{H}}=1.0,1.4$, 1.9 and $2.5 \mathrm{H} / \mathrm{Pd}$, respectively.
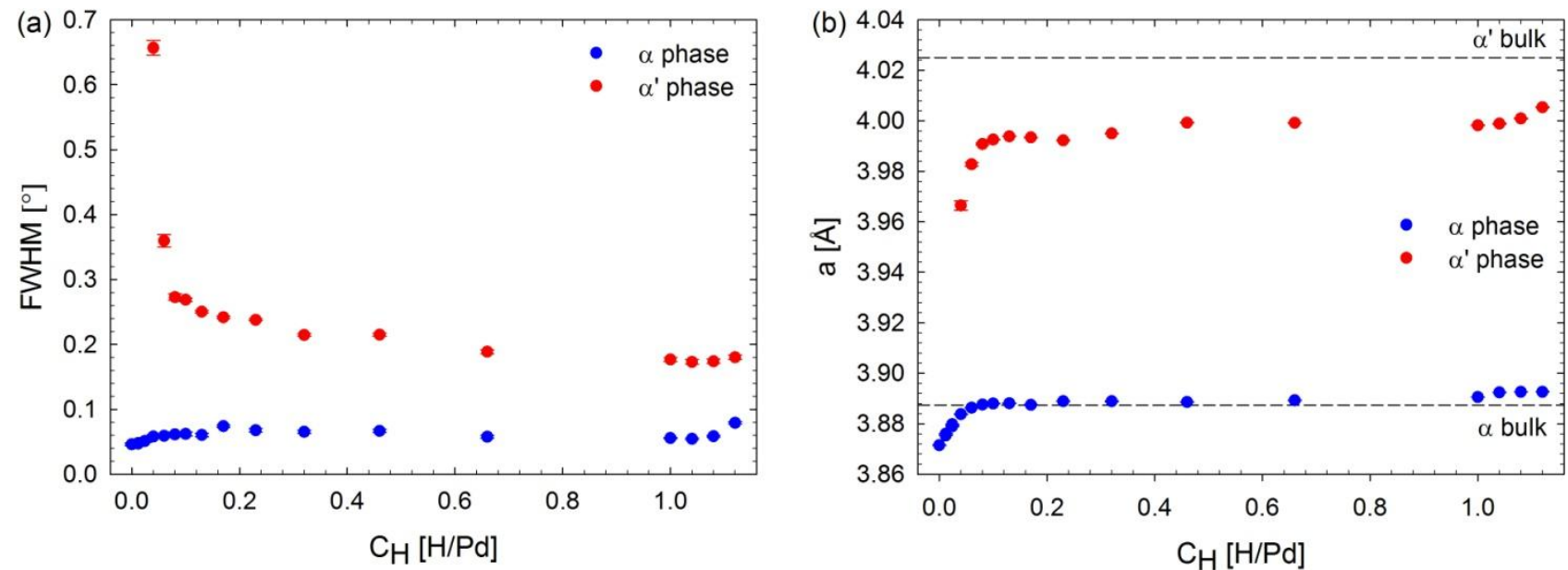

Figure 4 Epitaxial Pd film deposited on sapphire (1120) substrate: the development of (a) peak width and (b) lattice parameter during hydrogen loading. Dashed lines in the right panel shows the lattice parameter for the bulk $\alpha$ and $\beta$ phase reported in literature [6]. Data points grouped at $\mathrm{c}_{\mathrm{H}}=1.0 \mathrm{H} / \mathrm{Pd}$ represent nominal concentrations $\mathrm{c}_{\mathrm{H}}=1.0,1.4,1.9$ and $2.5 \mathrm{H} / \mathrm{Pd}$, respectively.

Results for epitaxial Pd films deposited on glimmer substrates are presented in Figs. 5 and 6. The development of the (111) peak for selected hydrogen concentrations plotted in Fig. 5a. It shows that the $(111)_{\alpha}$ peak firstly shifts to lower diffraction angles and then its position remains approximately constant with further loading. The $(111)_{\alpha^{\prime}}$ reflection appears at $\mathrm{c}_{\mathrm{H}}=0.045 \mathrm{H} / \mathrm{Pd}$ and its intensity 
increases with increasing hydrogen concentration due to $\alpha$ to $\alpha^{\prime}$ phase transition. The $(111)_{\alpha}$ peak decreases and completely disappears at $\mathrm{c}_{\mathrm{H}}>0.9 \mathrm{H} / \mathrm{Pd}$. Positions and widths of the (111) peaks plotted in Figs. 5a and 6b, respectively, behave in similar way as in the epitaxial film deposited on the sapphire substrate. The $(111)_{\alpha^{\prime}}$ peak firstly shifts to lower diffraction angles and then remains at approximately constant position. After its appearance at low hydrogen concentrations, the (111) $\alpha^{\prime}$ peak is rather broad. It becomes narrower at higher hydrogen concentrations. The width of the (111) $\alpha$ peak is virtually constant during the whole loading sequence.

The development of the out-of-plane lattice parameter for the $\alpha$ and the $\alpha^{\prime}$ phase is shown in Fig. 6b. The virgin film exhibits tensile in-plane stress similarly to the epitaxial film deposited on the sapphire substrate. During loading, the $\alpha$ phase lattice constant firstly increases and reaches a plateau close to the bulk value. After appearance of the $\alpha^{\prime}$ phase its lattice parameter increases and becomes saturated at a plateau value which is lower than the bulk value. Hence, the behavior of the epitaxial film deposited on glimmer is very similar to that for the epitaxial film grown on the sapphire substrate. But contrary to the epitaxial film grown on sapphire the epitaxial film deposited on glimmer buckles during the hydrogen loading. Slight dip in the lattice constant of $\alpha^{\prime}$ phase around $\mathrm{c}_{\mathrm{H}}=0.6 \mathrm{H} / \mathrm{Pd}$ might be caused by residual stress in buckles which disappears when films almost totally detach from the glimmer substrate at nominal concentration ' $\mathrm{c}_{\mathrm{H}}=0.9 \mathrm{H} / \mathrm{Pd}^{\prime}$.
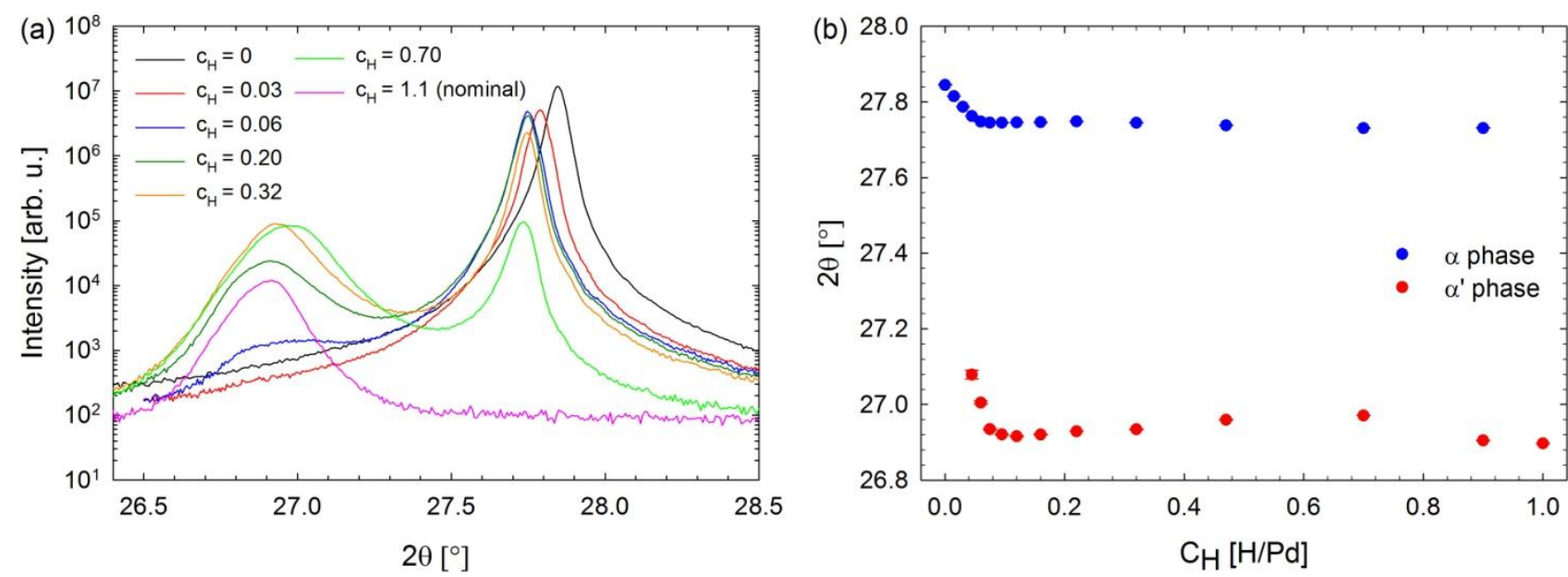

Figure 5 Epitaxial Pd film deposited on glimmer substrate: (a) the development of (111) peak for selected hydrogen concentrations; (b) the dependence of the peak position on hydrogen concentration.
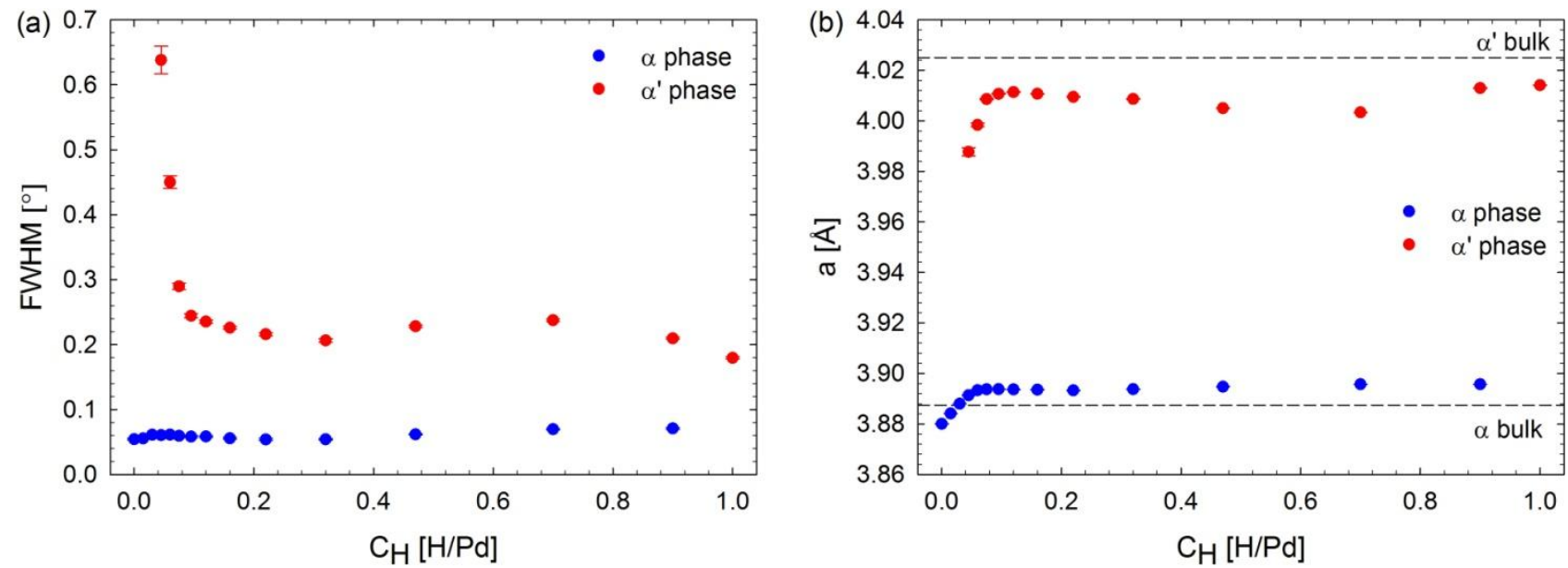

Figure 6 Epitaxial Pd film deposited on glimmer substrate: the development of (a) peak width and (b) lattice parameter during hydrogen loding. Dashed lines in the right panel shows the lattice parameter for the bulk $\alpha$ and $\beta$ phase reported in literature [6]. 


\section{Discussion}

The different microstructures of nanocrystalline and epitaxial films are accompanied by different stress states. The virgin nanocrystalline film exhibits compressive in-plane stress which is partly caused by the lattice constant mismatch between Pd and the sapphire substrate. Additional contribution to compressive in-plane stress comes from gas atom peening during the sputter deposition procedure [11]. Thereby, compressive stress is generated that cannot be released at room temperature.

The epitaxial films deposited at elevated temperature exhibit tensile in-plane stress. Stress generated by gas atom peening can be release at elevated temperature. But, because of the lower thermal expansion coefficient the thermal shrinkage of the sapphire substrate during cooling from elevated temperature is lower than that of Pd. This difference in the thermal expansion coefficient induces tensile stress into the epitaxial film.

As shown in the measurements, different microstructures lead to different behavior during the hydrogen loading. In the nanocrystalline film the $(111)_{\alpha^{\prime}}$ peak was firstly observed at $c_{H}=0.40$ $\mathrm{H} / \mathrm{Pd}$. Buckling and stress release occurs at $\mathrm{c}_{\mathrm{H}}=0.20 \mathrm{H} / \mathrm{Pd}$. This hints on the formation of $\alpha^{\prime}$ phase already at this hydrogen concentration, at least. It was recently shown that the $\alpha^{\prime}$ phase forms preferentially in the buckles and clamped parts of the film consist of $\alpha$ phase [12]. However, synchrotron XRD in Bragg-Brentano geometry is sensitive mainly to the clamped part of the film as (111) lattice planes in the buckles, except of a small part on the top of them, are bend away from the Bragg diffraction condition, see Fig. 7a. Thereby, the hydride related part of the sample becomes mainly invisible for XRD measurements and the final hydride peak intensity is very low. This may explain the relatively late direct detection of the $\alpha^{\prime}$ phase by appearance of the $(111)_{\alpha^{\prime}}$ peak in the diffractograms.

Nevertheless, $\alpha^{\prime}$ phase was observed at higher hydrogen concentrations than for the epitaxial films. There, hydride appeared already at $\mathrm{c}_{\mathrm{H}} \approx 0.04 \mathrm{H} / \mathrm{Pd}$. This difference is caused by the substantial volume fraction of grain boundaries in nanocrystalline films when compared to the epitaxial films. Grain boundaries are able to absorb more hydrogen than regular $\alpha$-phase crystallites. Hence, the concentration of hydrogen needed to form the $\alpha^{\prime}$ phase is increased by the amount of hydrogen stored at grain boundaries [13]. Detection of the $\alpha^{\prime}$ phase in epitaxial films was facilitated by the fact that both epitaxial films stayed clamped to the substrate in the concentration range where $\alpha^{\prime}$ phase was formed. Nominal hydrogen concentrations required for a complete hydride transformation of the nanocrystalline film deposited on sapphire and the epitaxial film deposited on glimmer were significantly higher than $\mathrm{c}_{\mathrm{H}}=0.60$ for the bulk Pd. This is caused by incomplete absorption of hydrogen during gas loading for high hydrogen concentrations because of hydrogen bubbles formation.

In the nanocrystalline film the lattice parameters of the $\alpha^{\prime}$ phase remain approximately constant during the $\alpha$ to $\alpha^{\prime}$ phase transition. But in the epitaxial films the $\alpha^{\prime}$ phase lattice parameter firstly increases and then, at higher hydrogen concentrations, it saturates at a constant value. The increase of the $\alpha^{\prime}$ phase lattice parameter is accompanied by narrowing of the $(111)_{\alpha^{\prime}}$ reflection. This indicates that the mechanism of the $\alpha^{\prime}$ phase formation differs in the nanocrystalline and epitaxial films. We suggest that in nanocrystalline films the $\alpha^{\prime}$ phase nucleates at the film surface and at grain boundaries. Thereby, $\alpha^{\prime}$ phase precipitates are formed also inside the $500 \mathrm{~nm}$ thick films. For epitaxial films the $\alpha^{\prime}$ phase most probably grows in thin layers starting from the film surface and gradually extends deeper and deeper into the $500 \mathrm{~nm}$ thick films. Schematic drawing of hydride growth in nanocrystalline and epitaxial films is shown in Fig. $7 b$. 
(a)

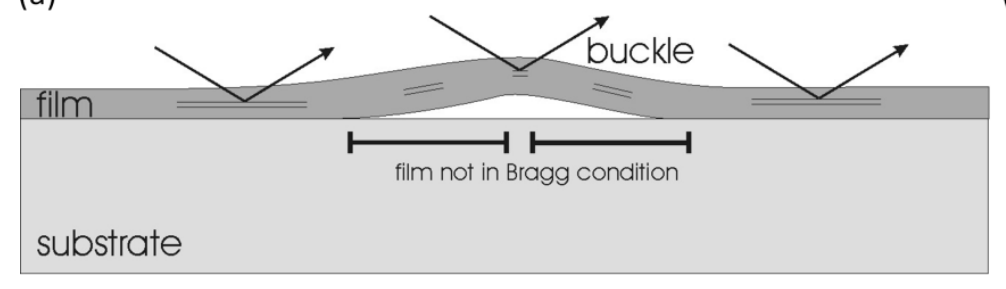

(b) Nanocrystalline film

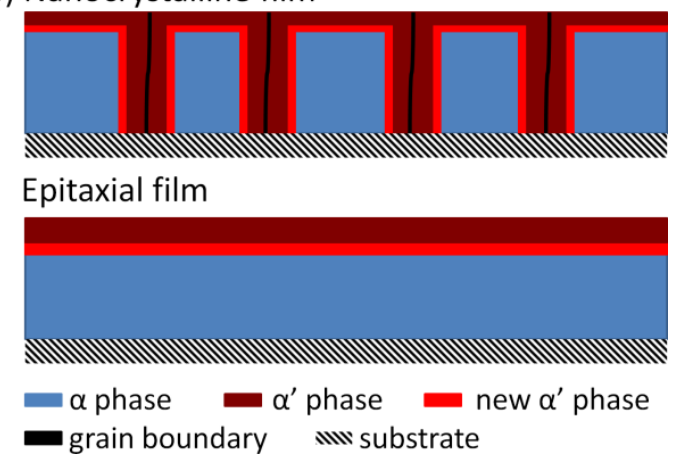

Figure 7 (a) Sketch of a film buckle showing the XRD Bragg visibility of the related film parts. (b) Sketch of a hydride growth in nanocrystalline and epitaxial Pd films.

A thin $\alpha^{\prime}$ phase layer formed at the surface in the beginning of the $\alpha$ to $\alpha^{\prime}$ phase transition explains a broad diffraction peak, as demonstrated in Figs. $4 \mathrm{a}$ and $6 \mathrm{a}$. The thin $\alpha^{\prime}$ phase layer has a coherent interface with the $\alpha$ phase and, therefore, suffers from in-plane stress imposed by the $\alpha$ phase Pd film beneath since minimization of the interface energy between the $\alpha^{\prime}$ phase layer and the Pd film is dominating factor in the early stages of the $\alpha$ to $\alpha^{\prime}$ phase transition. With increasing thickness of the $\alpha^{\prime}$ phase layer the bulk energy contribution becomes dominating and position of the $(111)_{\alpha^{\prime}}$ diffraction peak remains at a constant position. In addition to this the increasing thickness of the $\alpha^{\prime}$ phase layer makes the (111) $\alpha^{\prime}$ peak narrower, as found in Figs. 4a and 6a.

The behavior of both types of hydrogen loaded epitaxial Pd films is rather similar despite of the substrate used. But some differences between the epitaxial films deposited on sapphire and on glimmer were observed. These differences are obviously caused by the strength of the film adhesion to the substrate. It is higher for the film on sapphire than for the film on glimmer. Different adhesion strength is demonstrated by the fact that the film deposited on glimmer buckled during the hydrogen loading while the film grown on sapphire did not. Because of the higher adhesion of the epitaxial $\mathrm{Pd}$ film to the sapphire substrate $\left(\mathrm{W}_{\mathrm{adh}}=2.8 \mathrm{~J} / \mathrm{m}^{2}[14]\right)$ the film layer adjacent to the substrate suffers from very high stress which prevents forming of $\alpha^{\prime}$ phase. This is most probably the reason why the epitaxial film deposited on the sapphire substrate is not transformed to the $\alpha^{\prime}$ phase completely but the $(111)_{\alpha}$ peak was observed even after loading up to nominal concentration ${ }^{\prime} \mathrm{c}_{\mathrm{H}}=2.5 \mathrm{H} / \mathrm{Pd}^{\prime}$, see Fig. 3a. On the other hand, in epitaxial films deposited ion glimmer the stresses in the layer adjacent to the substrate are generally lower and are relaxed when the films detached from the substrate. This enables to transform the film into the $\alpha^{\prime}$ phase completely as demonstrated by the disappearance of the $(111)_{\alpha}$ peak in the XRD pattern.

\section{Summary}

Mechanism of growth of the $\beta$ phase in Pd films depends on the microstructure of the film. In nanocrystalline film the $\beta$ phase nucleates at the grain boundaries and is formed in the whole volume of the film while in the epitaxial films the $\beta$ phase growths in layers starting from the surface and gradually extending deeper into the film. High bonding strength of the epitaxial film deposited on sapphire substrate prevents buckling and inhibits complete transformation of the film to the $\beta$ phase. The epitaxial film deposited on glimmer substrate exhibits lower bonding strength and detaches from the substrate during hydrogen loading. This relaxes the stresses imposed by the substrate and allows complete transformation of the film to the $\beta$ phase.

\section{Acknowledgments}

This work was supported by the Grant Agency of Charles University (project no. 566012), the Czech Science Foundation (project no. P108/12/G043), the Deutsche Forschungsgemeinschaft DFG 
(projects PU131/9-1 and PU131/10-1) and Grant Vakuum Praha. Beamtime was kindly provided by the European Synchrotron Radiation Facility ESRF, Grenoble, at beamline BM20.

\section{References}

[1] U. Laudahn, A. Pundt, M. Bicker, U. Hülsen, U. Geyer, T. Wagner, R. Kirchheim: J. Alloy Compd. 293 (1999), p. 490

[2] A. Pundt, P. Pekarski: Scripta Mater. 48 (2003), p. 419

[3] Y. Yu, C. Kim, S.C. Sanday: Thin Solid Films 196 (1991), p. 229

[4] J. Č́ížek, I. Procházka, M. Vlach, N. Žaludová, S. Daniš, P. Dobroň, F. Chmelík, G. Brauer, W. Anwand, A. Mücklich, E. Nikitin, R. Gemma, R. Kirchheim, A. Pundt: Appl. Surf. Sci. 255 (2008), p. 241

[5] T.B. Flanagan, W.A. Oates: Annu. Rev. Mater. Sci. 21 (1991), p. 269

[6] F.D. Manchester, A. San-Martin, J.M. Pitre: J. Phase Equilib. 15 (1994), p. 62

[7] S. Wagner, H. Uchida, V. Burlaka, M. Vlach, M. Vlcek, F. Lukac, J. Cizek, C. Baehtz, A. Bell, A. Pundt: Scripta Mater. 64 (2011), p. 978

[8] J. Č́ížek, O. Melikhova, M. Vlček, F. Lukáč, M. Vlach, I. Procházka, W. Anwand, G. Brauer, A. Mücklich, S. Wagner, H. Uchida, A. Pundt: Int. J. Hydrogen Energ. 38 (2013), p. 12115

[9] R. Kirchheim: Prog. Mater. Sci. 32 (1988), p. 261

[10]S. Wagner, A. Pundt: Acta Mater. 58 (2010), p. 1387

[11]A. Debelle, G. Abadias, A. Michel, C. Jaouen: Appl. Phys. Lett. 84 (2004), p. 5034

[12]S. Wagner, M. Moser, C. Greubel, K. Peeper, P. Reichart, A. Pundt, G. Dollinger: Int. J. Hydrogen Energ. 38 (2013), p. 13822

[13]A. Pundt, R. Kirchheim: Annu. Rev. Mater. Res. 36 (2006), p. 555

[14]K. Højrup Hansen, T. Worren, S. Stempel, E. Lægsgaard, M. Bäumer, H.J. Freund, F. Besenbacher, I. Stensgaard: Phys. Rev. Lett. 83 (1999), p. 4120 